\section{Sharing: lessons from natural history's success story}

SIR - The success of data sharing among natural-history collections may alleviate the fears about capacity and cooperation expressed in your special issue (Nature 461, 145, 160-163 and 168-173; 2009). Our social and information-technology (IT) infrastructure provides open access to millions of records from hundreds of repositories, thanks to broad participation and funding from the US National Science Foundation. However, this success story also exposes some new challenges.

Key to this success has been the development of a distributed publishing system that conserves full rights of contributors to data and access. Data are curated at source and then made available to the community for use and improvement. The Mammal Networked Information System (MaNIS) was established in 2001 to provide hardware, data standards, transmission mechanisms and stakeholder support for open access to specimen data.

Since then, more thematic, taxonomic and regional networks have been created. Each of these feeds into a growing biodiversityinformatics community, including the Global Biodiversity Information Facility, which serves users with up to 189 million plant and animal records. The vertebrate-based biodiversity networks - including MaNIS, HerpNET, ORNIS and FishNet 2 serve some $4 \%$ of their combined holdings each day to users hungry for these data.

Sustaining these resources is difficult. Growth has led to problems with scalability and sustainability, including difficulties in keeping resources running, slow provider response times and complicated installations and maintenance. The National Biological Information Infrastructure has provided support, and the vertebrate networks are consolidating into a platform called VertNet. In order to reduce IT costs, VertNet will move from institutional servers to a cloud computing platform, providing nearly unlimited room for growth.

But solving technological challenges is not enough. Our success has depended on strong engagement with our contributor and user community. Capacity building in biodiversity informatics is especially important. Success requires willing participation, robust technology choices and a commitment to engage fully with the communities these repositories will serve.

Robert Guralnick University of Colorado Museum of Natural History, University of Colorado-Boulder, Boulder, Colorado 80309, USA e-mail: robert.guralnick@colorado.edu Heather Constable, John Wieczorek, Craig Moritz Museum of Vertebrate Zoology, University of CaliforniaBerkeley, Berkeley,

California 94720, USA

A. Townsend Peterson Natural History Museum and Biodiversity Research Center, University of Kansas, Lawrence, Kansas 66045, USA

\section{No final answers yet on sex determination in birds}

SIR - Readers of J. A. M. Graves's News \& Views article 'Sex determination: Birds do it with a Z gene' (Nature 461, 177-178; 2009) may be left with the impression that a particular gene, DMRT1 on the Z chromosome, is the master determinant of sex in birds. However, the case is far from closed.

Graves discusses the findings of Craig A. Smith and colleagues (Nature 461, 267-271; 2009).

These data support the view that DMRT1 is required for testis development in chickens. But as the authors state, "Although Z-linked DMRT1 is required for testis development, it is possible that another Z-linked gene lies upstream of this gene in the avian male-determining pathway ... It is also possible that a female determinant lies on the avian W sex chromosome".

It may turn out that DMRT1 does determine sex in birds. But as yet other possibilities remain open.

Asato Kuroiwa Laboratory of Animal Cytogenetics, Faculty of Science, Hokkaido University, Sapporo 060-0810, Japan

e-mail: asatok@ees.hokudai.ac.jp

\section{Toxicity testing by category for 30,000 chemicals?}

SIR - Thomas Hartung and

Costanza Rovinda in their Opinion article (Nature 460, 1080-1081; 2009) argue that the European Chemicals Agency has underestimated the impact of European Union (EU) legislation on animal testing by six times, and that some toxicity tests should be suspended. Unfortunately, pragmatic solutions for the implementation of reduced animal testing under the EU's REACH legislation - for registration, evaluation, authorization and restriction of chemicals - have not yet been formulated in a clear operational manner.

On the basis of the experience in the US High Production Volume Challenge Program, where nontesting approaches have been applied successfully, the goals for REACH can be formulated in a 'smart' manner. In this US programme, around $81 \%$ of the chemicals were included in a chemical category and new testing was proposed for fewer than $10 \%$ of the human-health and ecotoxicity end-point data needed (K. Van Leeuwen et al. SAR QSAR Environ. Res. 20, 207-220; 2009).

If the United States can waive testing for most of its industrial chemicals, Europe can strive for at least $50 \%$ reduction in animal testing over the next five years by applying a similar approach. This will allow us to move away from the slow, traditional, chemical-bychemical procedures.

Achieving regulatory acceptance for such approaches in Europe is the main challenge. REACH has to assess at least 30,000 chemicals in the next 11 years, an increase of a factor of 300 compared with the past 14 years (G. Schaafsma et al. Reg. Toxicol. Pharmacol. 53, 70-80; 2009). Hence, category approaches are the way forward.

Kees van Leeuwen, Gerwin Schaafsma TNO Quality for Life, PO Box 360, 3700 AJ, Zeist, The Netherlands e-mail: k.vanleeuwen@tno.nl

\section{Authors beware, and protect your online identity}

SIR - Goudarz Molaei is right to express concern in his Correspondence about simultaneous submission of manuscripts to different journals (Nature 461, 723; 2009). As a professional journal editor with more than 20 years' experience, I would like to highlight here a worrying new problem I recently encountered: duplicate submission arising from author impersonation.

Unfortunately, online submission and review systems inadvertently encourage this unwelcome activity. For example, a co-author or colleague may be given the corresponding author's account password in order to submit his or her manuscripts - perhaps because of the corresponding author's lack of time or unfamiliarity with file creation and uploading. These people are then able to change the author's accounts, including the passwords, and submit manuscripts in that person's name without their knowledge.

So, authors, be wary of who has access to your account. Keep a check on what's happening and change your password after files have been submitted. 